\title{
Treatment options for advanced urothelial cancer after progression on chemotherapy and immune checkpoint inhibitors: a literature review
}

\author{
Vadim S. Koshkin $^{1 \wedge}$, Appledene S. Osbourne ${ }^{2}$, Petros Grivas $^{3} \wedge$ \\ ${ }^{1}$ Division of Hematology/Oncology, Department of Medicine, University of California San Francisco, Helen Diller Family Comprehensive Cancer \\ Center, San Francisco, CA, USA; ${ }^{2}$ Department of Internal Medicine, UC Davis Medical Center, Sacramento, CA, USA; ${ }^{3}$ Division of Medical \\ Oncology, Department of Medicine, University of Washington School of Medicine, Seattle Cancer Care Alliance, Seattle, WA, USA \\ Contributions: (I) Conception and design: VS Koshkin, P Grivas; (II) Administrative support: VS Koshkin, P Grivas; (III) Provision of study materials \\ or patients: All authors; (IV) Collection and assembly of data: All authors; (V) Data analysis and interpretation: All authors; (VI) Manuscript writing: \\ All authors; (VII) Final approval of manuscript: All authors. \\ Correspondence to: Petros Grivas. Division of Medical Oncology, Department of Medicine, University of Washington School of Medicine, Seattle \\ Cancer Care Alliance, 1144 Eastlake Ave E, Mailstop: LG-465, Seattle, WA 98109, USA. Email: pgrivas@uw.edu.
}

Objective: To describe the current treatment landscape in advanced urothelial cancer (aUC)/metastatic urothelial cancer and in particular to review the relevant literature highlighting recent advances in the treatment of patients with aUC after progression on chemotherapy and immune checkpoint inhibitor (ICI).

Background: aUC is a very aggressive disease with poor outcomes. Over the past several years, its treatment landscape has seen significant advances with the approval of ICI and targeted agents, which have led to improved outcomes. The current standard of care for most patients with aUC involves platinumbased chemotherapy followed by ICI after progression or as switch maintenance therapy (if no progression after chemotherapy). Treatment of patients following progression on ICI is more challenging, but novel therapies have been approved, such as erdafitinib for tumors with fibroblast growth factor receptor 2 (FGFR2) or FGFR3 activating mutation or fusion (can also be used following progression on platinumbased chemotherapy), enfortumab vedotin (EV) and sacituzumab govitecan (SG) in an unselected patient population. Many other trials in this space are currently ongoing and other promising agents may also potentially become available in the future.

Methods: Narrative overview of the recent literature relevant to the treatment of advanced/metastatic urothelial cancer following progression on chemotherapy and ICI was undertaken. Relevant literature was obtained from review of computerized databases including pubmed.gov and proceedings of major conferences including American Society of clinical Oncology (ASCO) Meetings, GU ASCO Symposia and European Society of Medical Oncology (ESMO) Meetings.

Conclusions: In this narrative review, we highlight the current dynamic treatment landscape in aUC, emphasizing the recent important developments and a few examples of ongoing clinical trials. In particular, we focus on therapy options available following progression on platinum-based chemotherapy and ICI, a treatment space where until recently there had been no FDA-approved treatment options. The recent pivotal trials of antibody drug conjugates (ADCs) that led to FDA approvals in this space are highlighted, as are other agents currently in development. We conclude by discussing future directions and ongoing challenges in this evolving disease space.

Keywords: Advanced urothelial cancer (aUC); bladder cancer; immune checkpoint inhibitor (ICI); chemotherapy; targeted therapy

^ ORCID: Vadim S. Koshkin, 0000-0003-2277-8668; Petros Grivas, 0000-0003-3965-3394. 
Submitted Feb 08, 2021. Accepted for publication Jul 12, 2021.

doi: $10.21037 /$ tau-21-123

View this article at: https://dx.doi.org/10.21037/tau-21-123

\section{Introduction}

Bladder cancer is a common malignancy and is among the top ten most common tumors worldwide with almost 600,000 cases and almost 200,000 deaths in 2018 (1). The majority of patients are men and the average patient is in their late 60 s or early 70 s at the time of diagnosis. Clinical and pathologic staging plays a very important role in prognostication and management. Most patients are diagnosed with non-muscle invasive bladder cancer (NMIBC), however up to $25 \%$ have muscle-invasive bladder cancer (MIBC) at the time of diagnosis, implying the invasion of the bladder muscularis propria, with about $5 \%$ having distant metastatic spread at time of diagnosis. Most patients with bladder cancer have tumors with urothelial carcinoma histology, but up to $15-25 \%$ may have pure or mixed histological variants, including but not limited to: squamous cell, adenocarcinoma/glandular features, neuroendocrine/small cell, micropapillary, plasmacytoid, nested, sarcomatoid, among others. These tumors can arise anywhere in the genitourinary tract, which includes urethra, bladder (most common site), ureters and renal pelvis. Both histology and location also influence the prognosis and treatment considerations, and generally speaking, upper tract tumors are considered more aggressive relative to lower tract, and histological variants more aggressive relative to tumors with urothelial histology. While patients with NMIBC and localized MIBC are candidates for curative intent treatment, patients with distant metastases are generally considered incurable. Even in this treatment setting however, several treatment options are available and the recent years have brought about important new treatment considerations specifically for advanced urothelial cancer (aUC) (2). Several agents and combinations are also currently in the clinical trial pipeline as part of this dynamic landscape. However, the treatment of patients progressing on initial lines of therapy remains very challenging. This review highlights the novel treatment options in aUC, focusing in particular on the treatment options available following progression on chemotherapy and immune checkpoint inhibitors (ICI). We present the following review in accordance with the Narrative Review reporting checklist (available at https://dx.doi.org/10.21037/tau-21-123).

\section{Methods}

As part of this narrative literature review, we identified the relevant publications over the last 10 years in the English language related to the treatment of patients with advanced/metastatic urothelial cancer. Special focus was made on the literature related to the treatment of patients with aUC following progression on chemotherapy and ICI. Relevant literature was obtained from review of computerized databases including pubmed.gov, clinicaltrials. gov and proceedings of major conferences including American Society of clinical Oncology (ASCO) Meetings, Genitourinary ASCO Symposia and European Society of Medical Oncology (ESMO) Meetings.

\section{Current standard of care}

Cisplatin-based chemotherapy has been the standard of care for aUC since the 1980s. One of the initial trials to show clinical efficacy of the classic cisplatin combination regimen MVAC (methotrexate, vinblastine, doxorubicin, cisplatin) had an objective response rate (ORR) 69\% with complete responses (CRs) in $37 \%$, and $\mathrm{PD}$ as best response in only $23 \%$ (3). This combination regimen was shown to be superior to cisplatin monotherapy in a randomized prospective clinical trial with superior ORR (39\% vs. 12\%) and importantly superior mOS (12.5 vs. 8.5 months) (4). Subsequent clinical trials focused on the development of more tolerable regimens including dose-dense regimen (ddMVAC) administered in 2-week rather than 4-week cycles, along with G-CSF support (5). Additionally, a large randomized trial comparing the classic MVAC with gemcitabine/cisplatin (GC) in patients with aUC did show that GC was more tolerable with similar clinical outcomes including ORR, PFS and OS (6,7). The median OS in that trial was 15 months, with a 5 -year OS of $13-15 \%$, suggesting potential for long term responses in select patients treated with cisplatin-based chemotherapy (7). Consequently, the current NCCN guidelines recommend patients with aUC who are cisplatin-eligible to receive either GC or ddMVAC in the frontline setting. 
Eligibility for cisplatin-based treatment is defined according to the established Galsky criteria (8), and most patients are considered ineligible for cisplatin-based chemotherapy due to inadequate renal function (GFR $<60 \mathrm{~mL} / \mathrm{min}$ ) or poor PS ECOG $(>1)$. Patients with aUC ineligible to receive cisplatin have treatment options that include carboplatin-based chemotherapy or ICI. Carboplatin-based regimens can be used in patients with a moderately impaired renal function (GFR $30-60 \mathrm{~mL} / \mathrm{min}$ ) and are usually better tolerated than cisplatin-based regimens, however have historically been considered to be associated with inferior outcomes (9). Additionally, both pembrolizumab (anti-PD-1 agent) and atezolizumab (antiPD-L1 agent) have received accelerated FDA approval for the treatment of cisplatin-ineligible treatmentnaïve patients with aUC with ORR reported at 23-29\% in phase II single arm trials and CR rates approaching $10 \%$. Importantly these agents were generally much better tolerated than frontline cytotoxic chemotherapy $(10,11)$. A longer follow-up on the KEYNOTE-052 trial of frontline pembrolizumab alone showed median OS 11.3 months in all comers, but with higher ORR $47 \%$ and median OS 18.5 months in patients with higher PDL1 expression $(C P S \geq 10)(12,13)$. Moreover the clinical benefit was consistent regardless of age or performance status (including ECOG 2) (14).

The FDA issued a label restriction suggesting that only cisplatin-ineligible patients with tumors expressing high PD-L1 be treated with frontline ICI monotherapy. However, patients with aUC judged to be ineligible for any platinum-based treatment can receive ICI monotherapy without additional PD-L1 testing (in the US only) based on FDA guidance. Consequently, the current standard of care for patients with aUC ineligible for cisplatin-based chemotherapy is either carboplatin-based chemotherapy (with avelumab switch maintenance if no progression) or consideration of atezolizumab or pembrolizumab for patients whose tumors have high PD-L1 expression based on the corresponding companion assay for each agent. The cisplatin-ineligible front-line space remains an area of clinical need and numerous clinical trial combinations, including antibody drug conjugates (ADCs) and novel immunotherapy agents are being explored in this space $(15,16)$. Therefore, the treatment paradigm for treatmentnaïve cisplatin-ineligible patients might potentially shift in the future.

Two large phase III clinical trials have investigated the combination of chemotherapy and ICI for treatment- naïve patients with aUC in comparison with platinumbased chemotherapy alone. IMvigor 130 investigated the addition of atezolizumab to platinum-based chemotherapy $v s$. placebo/chemotherapy as frontline treatment in aUC, showing improvement in median PFS of 8.2 vs. 6.3 months (stratified HR $0.82, \mathrm{P}=0.007$ ) with no significant improvement in OS (16.0 vs. 13.4 months) at that time (17). The KEYNOTE-361 trial investigated the combination of frontline pembrolizumab with platinum-based chemotherapy $v s$. frontline chemotherapy alone, but did not meet its prespecified endpoints of PFS or OS improvement with the combination (18). The Danube trial assessed the combination of durvalumab and tremelimumab (in all comers), or durvalumab monotherapy (in the subset of PDL1-high tumors) $v s$. standard platinum-based chemotherapy. Neither comparison met its primary endpoint of OS benefit in the intention to treat population (19). Two other large phase III clinical trials combining frontline platinum-based chemotherapy with ICI are yet to report: checkmate-901 is comparing the combinations of nivolumab and ipilimumab, or nivolumab and GC, $v s$. platinum-based chemotherapy, while NILE is comparing combinations of durvalumab with chemotherapy, or durvalumab with tremelimumab with chemotherapy, vs. platinum-based chemotherapy. Additionally, the enfortumab vedotin-302 (EV-302) trial, building on the impressive results of the $\mathrm{EV} /$ pembrolizumab in the $\mathrm{EV}-103$ trial, is investigating the $\mathrm{EV} /$ pembrolizumab vs. platinum-based chemotherapy for treatment-naïve patients with aUC $(16,20)$.

For patients with aUC treated with 4-6 cycles of platinum-based chemotherapy, achieving response or stable disease, switch maintenance avelumab is now the new standard of care (level I evidence). This is based on the Javelin Bladder 100 trial which randomized patients who had no progression after frontline platinum-based chemotherapy to receive avelumab plus best supportive care or best supportive care alone, as maintenance therapy. With 350 patients randomized per arm, the median OS was significantly longer with avelumab (21.4 vs. 14.3 months; HR 0.69; $\mathrm{P}=0.001$ ) (21). A smaller randomized phase II trial of switch maintenance pembrolizumab vs placebo (with cross-over built in) showed a significant improvement in PFS for patients on pembrolizumab (median PFS 5.4 vs. 3.0 months, $\mathrm{P}=0.04$ ), but did not show significant OS benefit (22).

For patients who have progression on platinum-based chemotherapy, ICI or the fibroblast growth factor receptor (FGFR) inhibitor erdafitinib can be used as second line 
therapy. Initially five ICI were FDA-approved in this space: pembrolizumab, atezolizumab, nivolumab, avelumab and durvalumab; the FDA approvals were based mostly on data from several phase II trials, except for pembrolizumab (23-27). Recently, the post-platinum therapy applications for atezolizumab and durvalumab were voluntarily withdrawn. Only pembrolizumab is supported in this setting by level I evidence based on a randomized phase III trial that showed a significant OS advantage with pembrolizumab $v s$. salvage chemotherapy (docetaxel, paclitaxel or vinflunine) (median OS 10.3 vs. 7.4 months; HR 0.73, $\mathrm{P}=0.002$ ) (24). This benefit was maintained beyond two years of followup with superior 2-year OS rate for pembrolizumab relative to chemotherapy (27\% vs. 14\%) and a recently updated superior 3 -year OS rate $(21 \%$ vs. $11 \%)(28,29)$. The phase III IMvigor211 trial with atezolizumab vs. salvage chemotherapy did not meet its primary endpoint (30).

For a molecularly-selected subset of patients with aUC refractory to platinum-based chemotherapy, erdafitinib is a targeted agent with a novel mechanism of action that received accelerated FDA approval in April 2019. It is estimated that around $15-20 \%$ of patients with bladder cancer harbor FGFR3 alterations (31), which are further enriched in upper tract urothelial tumors (32). A single arm, open label phase II trial, BLC2001, assessed the efficacy and safety of erdafitinib in 99 patients with aUC who progressed on prior platinum-based chemotherapy or were cisplatin-ineligible if chemotherapy naïve (33). Patients had to have documented FGFR2 or FGFR3 mutation or fusion in the tumor. A significant minority of the patients in this trial (22\%) had also previously progressed on ICI. The confirmed ORR was $40 \%$, with $37 \%$ PRs and $3 \%$ CRs, while another $39 \%$ had SD as best response. Importantly, among patients who had previously progressed on ICI the response rate was $59 \%(13 / 22)$, making this an additional viable treatment option in patients who have previously progressed on both platinum-based chemotherapy and ICI. Based on the initial phase II trial data, erdafitinib was granted FDA accelerated approval for platinumrefractory aUC in April 2019, but a confirmatory phase III trial is pending. The THOR phase III trial is randomizing patients with FGFR3 activating mutation or fusion and progression on prior ICI to receive either erdafitinib or salvage chemotherapy (taxane or vinflunine) and patients with prior chemotherapy to receive either erdafitinib or pembrolizumab. Additionally the NORSE trial is investigating the combination of erdafitinib and cetrelimab (anti-PD-1) in patients who previously progressed on at least one systemic therapy, but not a prior FGFR or PD-L1 inhibitor (34).

\section{Recently approved treatments after progression on chemotherapy and ICI}

As discussed so far, the current standard of care for the majority of patients with aUC involves treatment with platinum-based chemotherapy followed by treatment with anti-PD-L1, either as switch-maintenance for patients who achieve response or stable disease, or as second-line treatment for patients with progression (Figure 1). However, nearly all patients with aUC will unfortunately progress on chemotherapy and on ICI. In the post-ICI space, there was no established standard of care until very recently. Salvage chemotherapy agents used in this space, such as paclitaxel, docetaxel or vinflunine could be expected to have limited responses with ORR $\sim 10-15 \%$ in this setting (35). Therefore, ADCs, such as EV and sacituzumab govitecan (SG), were developed to address that unmet need, while erdafitinib is also an option in that setting if not previously used, also based on the biomarker presence.

$\mathrm{EV}$ is an $\mathrm{ADC}$ that targets Nectin-4, which is a protein from the Nectin family that plays a role in cell adhesion and is highly expressed in aUC (36). EV is composed of an antibody targeting Nectin-4, which is conjugated to the potent microtubule disrupting chemotherapy agent MMAE. The interaction of the antibody portion of the ADC with Nectin-4 on the surface of the tumor cell leads to the internalization of the ADC complex into the tumor cell and the release of MMAE, which results in tumor cell death. EV was initially investigated in EV-101, a phase I dose escalation/expansion trial of Nectin-4-expressing solid tumors, including aUC that progressed on at least one prior chemotherapy regimen and/or prior ICI (37). Although initially Nectin-4 expression was a requirement for enrollment, the protocol was amended to remove it since the vast majority of patients exhibited high levels of Nectin-4 tumor staining by IHC. Patients were treated with escalating doses of $\mathrm{EV}$ up to $1.25 \mathrm{mg} / \mathrm{kg}$ on days $1,8,15$ of a 28-day cycle, and although the maximum tolerated dose was not reached, the recommended phase II dose (RP2D) was $1.25 \mathrm{mg} / \mathrm{kg}$. Overall, the treatment was relatively well tolerated with most common TRAEs including rash, peripheral neuropathy, fatigue, alopecia, and nausea. ORR among the 112 patients treated at the RP2D was $43 \%$ (5\% CR).

EV-201 was a phase II single arm study of EV in 


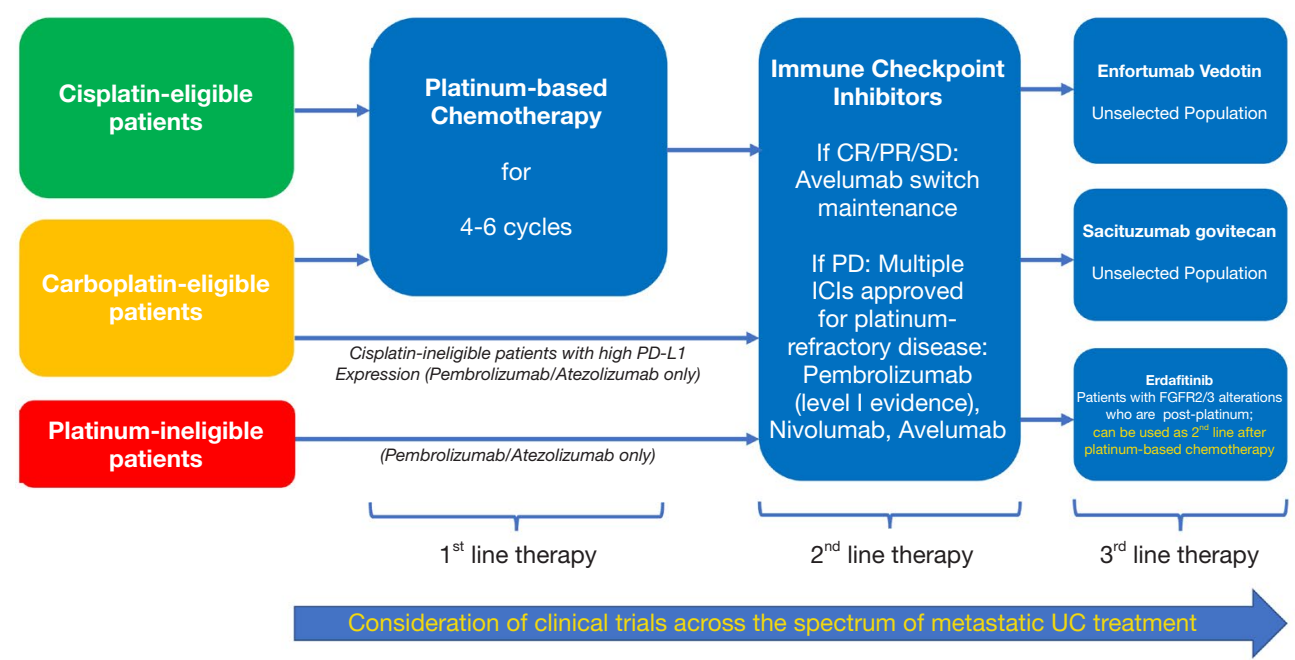

Figure 1 Current treatment landscape for patients with advanced urothelial cancer. CR, complete response; UC, urothelial carcinoma; ICI, immune checkpoint inhibitor; FGFR, fibroblast growth factor receptor.

patients with aUC divided in two cohorts (38). Cohort 1 enrolled patients who had received prior platinum-based chemotherapy and ICI, while cohort 2 enrolled patients whose disease had previously progressed on an ICI, but no prior chemotherapy given for aUC. The practice changing results from cohort 1 of EV-201 were published in the summer of 2019 (39). Among 125 patients, after a median follow-up of 10.2 months the confirmed ORR was $44 \%$ (12\% CR, 32\% PR) while another $28 \%$ of patients had SD as best response. The treatment was relatively well tolerated with most common TRAEs being fatigue, peripheral neuropathy, alopecia, and rash. Most TRAEs were $G 1$ or $G 2$ and no $\geq G 3$ events occurred in more than $10 \%$ of patients. Importantly, responses were again observed in key patient subsets, including patients with liver metastases (ORR $38 \%$ ), patients $\geq 75$ years of age (ORR $35 \%$ ) and patients with prior progression on ICI (ORR $41 \%)$. Updated data from cohort 1 was presented at the ESMO 2020 meeting, where after median follow-up of 22.3 months, the median OS was 12.4 months with $50.4 \%$ of patients being alive at 12 months and $34.2 \%$ alive at 18 months, respectively (40). These very encouraging results in the treatment-refractory setting led to FDA accelerated approval of EV in December 2019 for patients with aUC following progression on platinum-based chemotherapy and ICI.

This accelerated approval was contingent on the results of EV-301, the confirmatory phase III randomized trial comparing EV monotherapy with salvage chemotherapy (investigator choice of docetaxel, paclitaxel or vinflunine) in this setting. Recently published results of this trial reported superior outcomes with $\mathrm{EV}$ compared to salvage chemotherapy with significantly longer OS (HR 0.70; $\mathrm{P}=0.001)$ and PFS (HF 0.62; $\mathrm{P}<0.001)$ (41). Increasingly, real world data are emerging that also support the safety and efficacy of EV across a broad spectrum of patients. A multi-institutional retrospective study presented at the ESMO 2020 assessed the use of EV in an initial cohort of 83 patients and found an ORR $47 \%$, consistent with what was reported in trials. Importantly, responses were observed in patient subsets of interest who may have been excluded from clinical trials, such as patients with significant baseline neuropathy, diabetes mellitus regardless of its control, ECOG PS 2/3, or with significantly impaired renal function (42). Recently reported data from cohort 2 of the EV-201 trial also support the use of EV as second line therapy in patients previously treated with ICI, but not platinum-based chemotherapy. Among 89 patients, confirmed ORR was 52\% (95\% CI: 40.8-62.4), including $20 \%$ CR, median DOR about 11 months and mOS about 14.7 months (43). As of early 2021, EV has been established as a standard of care for patients with aUC who previously progressed on platinum-based regimen and ICI, and it appears that clinical benefit may extend also to patients who have not been previously treated with platinum-based chemotherapy. However, many questions remain, including optimal sequencing of this agent in the broader aUC treatment paradigm, toxicity mitigation/ 
control and potential predictive biomarkers. Other novel agents are also emerging in the post-ICI space.

\section{$S G$}

Among the most promising new investigational therapies for patients with aUC whose disease has progressed on prior ICI is another ADC, SG, previously known as IMMU-132. SG is composed of a humanized antibody directed against Trop-2, which is linked to $\mathrm{SN}-38$, an active metabolite of irinotecan. Trophoblast cell-surface antigen 2 (Trop-2) is a trans-membrane protein involved in several signaling pathways associated with tumor growth, invasion and spread, which is overexpressed on many epithelial tumors including urothelial carcinoma. Higher expression of Trop-2 has been linked to poor prognosis. In UC, increased expression of Trop-2 has been indeed correlated with cancer severity (44).

SG was originally investigated in a first-in-human trial of 25 patients with a variety of epithelial tumors who had experienced progression on conventional treatments (45). Four different dose levels of 8, 10, 12 and $18 \mathrm{mg} / \mathrm{kg}$ were administered on days 1 and 8 of a 21-day cycle, and patients were not pre-selected based on Trop-2 expression levels. The agent was found to have acceptable toxicity overall with neutropenia being the dose limiting adverse event, while 8 and $10 \mathrm{mg} / \mathrm{kg}$ doses were selected for phase II trials. Subsequent results of the aUC cohort from the phase I/ II basket trial included 41 patients treated at the RP2D of $10 \mathrm{mg} / \mathrm{kg}$ (46). These were pre-treated patients with a median of three prior therapies who received a median of 12 doses of SG as part of this trial. ORR was $34 \%$ (1 CR, 13 PRs) in the overall population and importantly $39 \%$ in patients with liver metastases. The median time to response was 1.9 months and median DOR was 12.9 months. Most common grade $\geq 3$ adverse events included neutropenia (28\%), fatigue (9\%), anemia (9\%) and diarrhea (6\%). Updated data from this trial presented in 2019 reported the confirmed ORR at $31 \%$ and median PFS and OS to be 7.3 and 18.9 months, respectively among 45 patients (47). These promising results led to the development of a global multi-cohort phase II trial, TROPHY-U-01 (48).

TROPHY-U-01 (NCT03547973) was a singlearm, phase II trial evaluating the antitumor activity of SG $(10 \mathrm{mg} / \mathrm{kg}$ on days 1 and 8 of a 21 -day cycle) in 140 patients with aUC (49). Cohort 1 was the pivotal cohort including patients who progressed on both platinumbased chemotherapy and ICI and aimed to enroll 100 patients. Cohort 2 included platinum-ineligible patients who progressed on ICI and is aiming to enroll 40 patients. Most recently, an updated analysis of cohort 1 , including 113 evaluable patients, was presented at the ESMO 2020 meeting. The ORR based on central review was $27 \%$ (31/113) with 6 CRs and 25 PRs, while the clinical benefit rate was $37 \%(42 / 113)$. Median duration of response was 5.9 months, whereas median PFS and OS were 5.4 months and 10.5 months, respectively. Toxicities were consistent with previously reported results with most common grade $\geq 3$ adverse events included neutropenia $(35 \%)$, anemia $(14 \%)$, febrile neutropenia $(10 \%)$ and diarrhea $(10 \%)$. One treatment-related death from neutropenic sepsis was reported. Preliminary results from Cohort 2 of TROPHY-U-01 were also presented at the ASCO 2020 meeting (50). Among 18 evaluable patients, with a median follow-up of 6 months the ORR was $28 \%$ (5/18, all PRs) and most patients did have reduction in target lesions. Most common adverse events included cytopenias, particularly neutropenia, fatigue and diarrhea. Cohort 3 of TROPHY-U-01 which combines SG with pembrolizumab in aUC previously progressed on platinumbased chemotherapy is currently enrolling (51). Further cohorts from this trial will also investigate combinations of SG in the treatment-naïve setting. Based on the initial promising data from cohort $1, \mathrm{SG}$ was granted accelerated FDA approval in April 2021 for patients with aUC following progression on platinum-based chemotherapy and ICI. TROPICS-04 (NCT04527991) is the ongoing confirmatory large phase III trial randomizing patients who progressed on platinum-based chemotherapy and ICI to receive SG or a chemotherapy agent of investigator's choice (docetaxel, paclitaxel or vinflunine) (52).

With both EV and SG now available as treatment options in the post-platinum and post-ICI third-line space, potential future questions will revolve around the optimal sequencing of these agents as well as potential biomarkers predicting response to either agent. Due to the largely non-overlapping toxicities and mechanism of action of these two ADCs, potential treatment decision between the two drugs could be based on efficacy, presence of existing medical comorbidities (e.g., severe peripheral neuropathy that can be important in the context of prior treatment), toxicity profiles, level of evidence, provider comfort, patient preference and insurance coverage.

\section{Other agents and molecularly selected therapies}

A number of other novel agents are also being investigated 
in aUC refractory to ICI. ASG-15ME is another ADC that targets SLITRK6 with a payload of MMAE chemotherapy agent, similar to EV (53). SLITRK6 is a type I transmembrane protein that can be highly expressed in bladder cancer. Initial activity and safety of ASG-15ME was investigated in a phase I dose escalation trial of 51 patients with aUC progressing on at least one prior chemotherapy regimen, with six different dose levels studied (54). The ORR was $33 \%$, including robust ORR 42\% (5/12 patients) who had previously progressed on ICI. The agent was considered well tolerated with the most common TRAE being fatigue in $44 \%$ of patients, however no follow-up data with this drug have been presented to date in aUC.

Many clinical trials in aUC-and thus potential future treatment options-are molecularly driven, selecting patients with alterations commonly found in aUC for specific targeted treatments. The approval of erdafitinib for FGFR2/3-altered aUC was discussed above and has validated this approach. Overexpression of HER2, a transmembrane receptor involved in cell proliferation, and commonly also found in breast and gastric cancers, is another alteration commonly found in aUC for which multiple treatments have been considered. Trastuzumab deruxtecan (DS-8201a) is an ADC consisting of an antibody targeting HER2 linked to a topoisomerase inhibitor. This drug recently received FDA accelerated approval for patients with metastatic HER2-positive breast cancer and is being investigated in a phase $\mathrm{Ib}$ trial in aUC in combination with nivolumab in patients previously progressing on chemotherapy (55). Another ADC targeting HER2 is RC48-ADC, which is comprised of another HER2 targeting antibody, hertuzumab, which is conjugated with MMAE. This agent was initially assessed in a phase I basket trial of multiple solid malignancies and administered every 2 weeks. The drug was well tolerated with most common side effects including cytopenias and liver function test elevation. The ORR was $33 \%$ in evaluable patients and one of the partial responses was recorded in a patient with aUC (56). Based on the initial results, a phase II dose expansion trial was pursued at a dose of $2 \mathrm{mg} / \mathrm{kg}$ every 2 weeks in aUC previously treated with at least one systemic therapy. All eligible patients had HER2-positive tumors (IHC 2/3+). Among 43 patients, $18.6 \%$ had prior ICI; the ORR was $60.5 \%$ in the overall population and $75 \%$ in those previously treated with ICI, while TRAEs were consistent with previously reported data $(57,58)$. Another trial of RC48-ADC in patients with aUC following progression on platinum, gemcitabine and taxane was presented at the
2021 ASCO annual meeting (NCT03809013). This trial completed enrollment in September 2020 and included a total of 64 patients with HER2-overexpressing urothelial tumors (IHC $2+$ or $3+$ ). The independently assessed ORR was $46.9 \%$ and median OS was 14.8 months (59). Importantly, among 19 patients who had prior ICI, ORR was $42 \%$; ORR in patients with visceral metastases was $45 \%$. TRAEs were consistent with previously reported data.

PRS-343 is another novel bispecific antibody targeting HER2 and CD137 (4-1BB), a costimulatory molecule playing an important role in immune regulation (60). CD137 protein is part of the TNF receptor family that is expressed on several important immune cell subtypes, such as $\mathrm{CD}^{+}$and $\mathrm{CD}^{+}{ }^{+}$T-cells, B cells and NK cells (61). An update of the phase I dose escalation trial of PRS-343 in patients with advanced HER2+ solid tumors was recently presented at the ESMO 2020 meeting (62). Among 70 patients enrolled, 33 were treated at active dose levels and evaluable for response, although there were only two patients with aUC. The ORR and DCR were $12 \%$ and $52 \%$, respectively, but were associated with higher dose levels, while post-treatment expansion of $\mathrm{CD}^{+} \mathrm{T}$-cells was also observed. The treatment was well tolerated with most common TRAEs $(>5 \%)$ related to mild infusion reaction, nausea, and arthralgia. The combination of PRS-343 and atezolizumab is also being pursued in a phase I clinical trial of patients with HER2-positive solid tumors including aUC (NCT03650348).

Epigenetic modifiers are another important group of agents currently being investigated in aUC, as chromatinmodifying gene alterations are commonly found in bladder cancer (63). In particular, combinations of epigenetic modifiers with ICI are of interest given the hypothesis that novel tumor antigens may be exposed with this treatment. A phase I/II trial combining the epigenetic modifier guadecitabine with atezolizumab (NCT03179943) in patients with ICI-refractory aUC completed enrollment with results pending soon. Similar phase I trials investigating other epigenetic modifiers, such as abexinostat/ pembrolizumab combination (NCT03590054) are also ongoing. Multiple other classes of agents have also been investigated in aUC sometimes with disappointing results. In one such example, the poly (ADP-ribose) polymerase (PARP) inhibitor rucaparib was investigated as single agent in the ATLAS phase II trial, which included a molecularly unselected population with the majority of patients having received both platinum-based chemotherapy and ICI. No confirmed responses were seen in this setting (64). 
Table 1 Selected clinical trials in advanced urothelial cancer following progression on PD-L1 treatment

\begin{tabular}{|c|c|c|c|c|c|}
\hline Clinical trial & Phase & Treatment & Treatment setting & ORR (\%) & NCT number \\
\hline EV-201 (cohort 1) & II & Enfortumab vedotin & aUC s/p chemo/ICl & 44 & NCT03219333 \\
\hline EV-201 (cohort 2) & II & Enfortumab vedotin & $\begin{array}{c}\text { aUC s/p ICI (cisplatin- } \\
\text { ineligible) }\end{array}$ & 52 & NCT03219333 \\
\hline $\begin{array}{l}\text { SG phase I/II basket } \\
\text { study (UC cohort) }\end{array}$ & $\mid / I I$ & Sacituzumab govitecan & $\begin{array}{c}\text { aUC s/p } \geq 1 \text { therapy; }(95 \% \\
\text { chemo, } 48 \% \text { ICl) }\end{array}$ & 31 (post-ICl 23) & NCT03547973 \\
\hline TROPHY-U-01 (cohort 1) & ॥ & Sacituzumab govitecan & aUC s/p chemo/ICl & 27 & NCT03547973 \\
\hline TROPHY-U-01 (cohort 2) & II & Sacituzumab govitecan & $\begin{array}{c}\text { aUC s/p ICI (cisplatin- } \\
\text { ineligible) }\end{array}$ & 28 & NCT03547973 \\
\hline $\begin{array}{l}\text { RC48-ADC phase II } \\
\text { urothelial study }\end{array}$ & ॥ & RC48-ADC & $\begin{array}{c}\mathrm{HER} 2 \text { + aUC patients s/p } \\
\text { platinum and } \mathrm{ICl}(19 \%)\end{array}$ & 51 ; post-IO 75 & NCT03507166 \\
\hline $\begin{array}{l}\text { RC48-ADC phase II } \\
\text { urothelial study }\end{array}$ & ॥ & RC48-ADC & $\begin{array}{l}\text { HER2 + aUC patients s/ } \\
\text { p platinum-based regimen } \\
\text { and taxane; ICI (30\%) }\end{array}$ & 47 ; post-IO 42 & NCT03809013 \\
\hline
\end{tabular}

*, ORR in the enfortumab vedotin arm of EV-301. ORR, objective response rate; EV, enfortumab vedotin; aUC, advanced urothelial carcinoma; ICI, immune checkpoint inhibitor; chemo, chemotherapy; SG, sacituzumab govitecan; UC, urothelial carcinoma; ADC, antibody drug conjugate.

A summary of selected clinical trials in aUC following progression on ICI is presented in Table 1.

Other agents with potential activity in aUC include vascular endothelial growth factor receptor antagonists such as ramucirumab, or multikinase inhibitors including cabozantinib. Ramucirumab was investigated in the postplatinum space as part of the phase III RANGE trial which randomized 530 patients with aUC to receive docetaxel with ramucirumab or docetaxel with placebo and allowed prior treatment with ICI. Adding ramucirumab to docetaxel led to a statistically significant improvement in PFS [4.1 vs. 2.8 months; HR 0.696 (95\% CI: 0.573-0.845), $\mathrm{P}<0.001$ ], but no significant improvement in OS [9.4 vs. 7.9 months; HR 0.887 (95\% CI: 0.724-1.086), $\mathrm{P}=0.25$ ] (65). Activity of cabozantinib was assessed as part of a phase II study in patients with platinum-refractory aUC. Among 42 postplatinum patients with aUC evaluable for response, ORR was $19 \%$ indicating single-agent activity (66). With strong pre-clinical rationale of combining cabozantinib with ICI, a phase I trial investigated the combination of cabozantinib with nivolumab or with nivolumab and ipilimumab in patients with aUC either naïve or refractory to ICI (67). The combination of cabozantinib with ICIs demonstrated manageable toxicities and reported responses in 5 out of 13 patients with aUC, thus suggesting potential benefit of adding cabozantinib to ICI treatment to overcome resistance to immunotherapeutic agents in this space (68).

\section{Future directions}

ICIs have profoundly altered the treatment landscape in aUC over the past several years, and now form the backbone for the standard of care, as either frontline treatment in platinum-ineligible patients, post-platinum switch maintenance treatment, or treatment for platinumrefractory disease. Increasingly, ICIs are moving into earlier treatment setting trials as well. In the neoadjuvant space, very promising phase II trials have shown compelling pathologic CR rates supporting five ongoing phase III perioperative trials in localized MIBC (69,70). Phase II trials of ICI and combinations of chemotherapy plus ICI have shown initial promising results (71-74). In the pure adjuvant space, 
Table 2 Examples of treatments for advanced urothelial cancer tested in various clinical trials

\begin{tabular}{lcc}
\hline Conventional cytotoxic agents & Immunotherapy & Targeted therapies \\
\hline Chemotherapy & Checkpoint inhibitors & Anti-angiogenesis \\
Antibody-drug conjugates & Vaccines & FGFR inhibitors \\
Radiation & Cytokines & HER family inhibitors \\
& Adoptive cell-based therapy & PARP inhibitors \\
& Other immuno-modulating agents & Chromatin remodeling (i.e., HDAC inhibitors) \\
& & Other (i.e., monoclonal antibodies, TKIs) \\
\hline
\end{tabular}

FGFR, fibroblast growth factor receptor; HER, human epidermal growth factor receptor; PARP, poly (ADP-ribose) polymerase; HDAC, histone deacetylase; TKI, tyrosine kinase inhibitor.

large randomized clinical trials have investigated the role of ICIs for high-risk disease at the time of radical surgery. While the IMvigor010 trial did not show significant benefit of adjuvant atezolizumab $v s$. observation (75), recently reported Checkmate-274 trial did show a significant disease-free survival benefit of adjuvant nivolumab $v s$. placebo in a similar patient population (76). The results of the AMBASSADOR trial of adjuvant pembrolizumab vs. observation and PROOF-302 (infigratinib vs. placebo) are still pending. For patients with BCG-unresponsive NMIBC who cannot undergo or refuse radical cystectomy, pembrolizumab is FDA-approved based on the results of Keynote-057 single arm phase II trial, while atezolizumab has also shown results in this treatment space (S1605 trial) without having FDA approval $(77,78)$.

Increasingly ICI-based combinations are being tested into the frontline space as well, particularly for the cisplatin-ineligible population, which is in especially high need of novel therapies. As part of the EV-103 clinical trial, the cohort of treatment-naive patients treated with combination of pembrolizumab plus EV showed a robust response rate of $73 \%$, leading to a randomized cohort of pembrolizumab/EV vs. EV to be enrolled in that same trial (16). Other trials of molecularly selected patients in the frontline space are also ongoing. This includes patients with low PD-L1 status being enrolled into the PIVOT-10 trial of combination nivolumab with NKTR-214 (pegylated IL-2) (15), while the LEAP-011 trial is testing the combination of pembrolizumab with either lenvatinib or placebo (79). For patients with FGFR3-altered tumors a number of trials are investigating the combination of ICIs with FGFR3 inhibitors $(34,80,81)$.

Despite these very promising developments and novel directions, the unfortunate reality remains that many patients with aUC may not respond to ICI, and nearly all patients eventually develop resistance and have tumor progression. Many patients are too sick or frail to tolerate third or fourth-line therapy, therefore decisions regarding treatments earlier in their disease course become more critical (82). The arsenal of currently available and possible future treatment options in aUC has expanded substantially (Table 2), but much remains to be learned in this challenging disease space. Important questions remain about resistance mechanisms to ICI and the optimal selection of patients for ICI monotherapy or therapy intensification. Significant research looking at prognostic and predictive biomarkers is underway and over the next few years may shed additional light on these very important questions.

\section{Conclusions}

The treatment landscape of aUC has seen dramatic changes over the past several years and remains a rapidly changing and dynamic space. The current treatment paradigm for most patients involves treatment with platinum-based chemotherapy followed by anti-PD-L1 agents as either switch maintenance therapy or as treatment for platinumrefractory disease. At the time of progression on $\mathrm{PD}$ L1 therapy, novel treatment options have also recently emerged. For molecularly-selected patients whose tumors harbor FGFR2 or FGFR3 activating mutation or fusion, erdafitinib is a treatment option in the platinumrefractory space and appears to also be effective for patients progressing on prior ICI. For an unselected population of patients with prior progression on platinum-based chemotherapy and ICI, EV (ADC targeting Nectin-4), and SG (ADC targeting Trop-2) are great treatment options. A number of other experimental therapies are also being 
developed in the post-ICI treatment space with very promising data. Increasingly novel therapies are also being developed for other molecularly selected populations, such as HER2 overexpression, including ADCs and bispecific antibodies, while combinations of either anti-VEGF or anti-FGFR agents with ICI look promising. Although significant advances in the treatment of platinum-refractory aUC have been made with several agents approved in this setting since 2016, this remains an aggressive and generally incurable disease. Further research focusing on novel treatment combinations as well as mechanisms of resistance and biomarkers of response will continue advancements in the field that are so urgently needed to help more patients.

\section{Acknowledgments}

Funding: None.

\section{Footnote}

Provenance and Peer Review: This article was commissioned by the Guest Editor (Tilman Todenhöfer) for the series "Management of Advanced Genitourinary Malignancies" published in Translational Andrology and Urology. The article has undergone external peer review.

Reporting Checklist: The authors have completed the Narrative Review reporting checklist. Available at https:// dx.doi.org/10.21037/tau-21-123

Conflicts of Interest: All authors have completed the ICMJE uniform disclosure form (available at https://dx.doi. org/10.21037/tau-21-123). The series "Management of Advanced Genitourinary Malignancies" was commissioned by the editorial office without any funding or sponsorship. Dr. PG serves as an unpaid editorial board member of Translational Andrology and Urology from Jul 2018 to Jun 2020. Dr. VSK reports outside funding for the institution from Nektar, Janssen, Clovis, and Endocyte, personal fees from Pfizer, Janssen, AstraZeneca, Dendreon, Clovis, and Seattle Genetics/Astellas, outside the submitted work. In the last 3 years (unrelated to this manuscript), Dr. PG has provided consulting to AstraZeneca, Bayer, Bristol Myers Squibb, Clovis Oncology, Dyania Health, Driver, EMD Serono, Exelixis, Foundation Medicine, Genentech/ Roche, Genzyme, GlaxoSmithKline, Heron Therapeutics, Immunomedics/Gilead, Infinity Pharmaceuticals, Janssen, Merck \& Co., Mirati Therapeutics, Pfizer, Regeneron
Pharmaceuticals, QED Therapeutics, Seattle Genetics, 4D Pharma PLC; his institution has received research funding from Bavarian Nordic, Bristol Myers Squibb, Clovis Oncology, Debiopharm, GlaxoSmithKline, Immunomedics, Kure It Cancer Research, Merck \& Co., Mirati Therapeutics, Pfizer, QED Therapeutics. Dr. ASO has no other conflicts of interest to declare.

Ethical Statement: The authors are accountable for all aspects of the work in ensuring that questions related to the accuracy or integrity of any part of the work are appropriately investigated and resolved.

Open Access Statement: This is an Open Access article distributed in accordance with the Creative Commons Attribution-NonCommercial-NoDerivs 4.0 International License (CC BY-NC-ND 4.0), which permits the noncommercial replication and distribution of the article with the strict proviso that no changes or edits are made and the original work is properly cited (including links to both the formal publication through the relevant DOI and the license). See: https://creativecommons.org/licenses/by-nc-nd/4.0/.

\section{References}

1. Bray F, Ferlay J, Soerjomataram I, et al. Global cancer statistics 2018: GLOBOCAN estimates of incidence and mortality worldwide for 36 cancers in 185 countries. CA Cancer J Clin 2018;68:394-424.

2. Koshkin VS, Grivas P. Emerging Role of Immunotherapy in Advanced Urothelial Carcinoma. Curr Oncol Rep 2018;20:48.

3. Sternberg CN, Yagoda A, Scher HI, et al. M-VAC (methotrexate, vinblastine, doxorubicin and cisplatin) for advanced transitional cell carcinoma of the urothelium. J Urol 1988;139:461-9.

4. Loehrer PJ Sr, Einhorn LH, Elson PJ, et al. A randomized comparison of cisplatin alone or in combination with methotrexate, vinblastine, and doxorubicin in patients with metastatic urothelial carcinoma: a cooperative group study. J Clin Oncol 1992;10:1066-73.

5. Sternberg CN, de Mulder PH, Schornagel JH, et al. Randomized phase III trial of high-dose-intensity methotrexate, vinblastine, doxorubicin, and cisplatin (MVAC) chemotherapy and recombinant human granulocyte colony-stimulating factor versus classic MVAC in advanced urothelial tract tumors: European Organization for Research and Treatment of Cancer 
Protocol no. 30924. J Clin Oncol 2001;19:2638-46.

6. von der Maase H, Hansen SW, Roberts JT, et al. Gemcitabine and cisplatin versus methotrexate, vinblastine, doxorubicin, and cisplatin in advanced or metastatic bladder cancer: results of a large, randomized, multinational, multicenter, phase III study. J Clin Oncol 2000;18:3068-77.

7. von der Maase H, Sengelov L, Roberts JT, et al. Longterm survival results of a randomized trial comparing gemcitabine plus cisplatin, with methotrexate, vinblastine, doxorubicin, plus cisplatin in patients with bladder cancer. J Clin Oncol 2005;23:4602-8.

8. Galsky MD, Hahn NM, Rosenberg J, et al. Treatment of patients with metastatic urothelial cancer "unfit" for Cisplatin-based chemotherapy. J Clin Oncol 2011;29:2432-8.

9. Galsky MD, Chen GJ, Oh WK, et al. Comparative effectiveness of cisplatin-based and carboplatin-based chemotherapy for treatment of advanced urothelial carcinoma. Ann Oncol 2012;23:406-10.

10. Balar AV, Galsky MD, Rosenberg JE, et al. Atezolizumab as first-line treatment in cisplatinineligible patients with locally advanced and metastatic urothelial carcinoma: a single-arm, multicentre, phase 2 trial. Lancet 2017;389:67-76.

11. Balar AV, Castellano D, O'Donnell PH, et al. First-line pembrolizumab in cisplatin-ineligible patients with locally advanced and unresectable or metastatic urothelial cancer (KEYNOTE-052): a multicentre, single-arm, phase 2 study. Lancet Oncol 2017;18:1483-92.

12. Vuky J, Balar AV, Castellano D, et al. Long-Term Outcomes in KEYNOTE-052: Phase II Study Investigating First-Line Pembrolizumab in CisplatinIneligible Patients With Locally Advanced or Metastatic Urothelial Cancer. J Clin Oncol 2020;38:2658-66.

13. O'Donnell PH, Balar AV, Vuky J, et al. First-line pembrolizumab (pembro) in cisplatin-ineligible patients with advanced urothelial cancer (UC): Response and survival results up to five years from the KEYNOTE-052 phase 2 study. J Clin Oncol 2021;39:abstr 4508.

14. Grivas P, Plimack ER, Balar AV, et al. Pembrolizumab as First-line Therapy in Cisplatin-ineligible Advanced Urothelial Cancer (KEYNOTE-052): Outcomes in Older Patients by Age and Performance Status. Eur Urol Oncol 2020;3:351-9.

15. Huddart RA, Siefker-Radtke AO, Balar AV, et al. PIVOT-10: Phase II study of bempegaldesleukin plus nivolumab in cisplatin-ineligible advanced urothelial cancer. Future Oncol 2021;17:137-49.

16. Rosenberg JE, Flaig TW, Friedlander T W, et al. Study EV-103: Preliminary durability results of enfortumab vedotin plus pembrolizumab for locally advanced or metastatic urothelial carcinoma. J Clin Oncol 2020;38:abstr 441.

17. Galsky MD, Arija JÁA, Bamias A, et al. Atezolizumab with or without chemotherapy in metastatic urothelial cancer (IMvigor130): a multicentre, randomised, placebocontrolled phase 3 trial. Lancet 2020;395:1547-57.

18. Alva A, Csoszi T, Ozguroglu M, et al. Pembrolizumab (P) combined with chemotherapy $(\mathrm{C})$ vs $\mathrm{C}$ alone as firstline (1L) therapy for advanced urothelial carcinoma (UC): KEYNOTE-361. Ann Oncol 2020;31:S1155.

19. Powles TB, Van Der Heijden MS, Gauna DC, et al. 6970 A phase III, randomized, open-label study of firstline durvalumab (D) with or without tremelimumab (T) vs standard of care chemotherapy in patients with unresectable, locally advanced or metastatic urothelial carcinoma (DANUBE). Ann Oncol 2020;31:S550-1.

20. Van der Heijden MS, Gupta S, Galsky MD, et al. 798TiP study EV-302: a 3-arm, open-label, randomized phase III study of enfortumab vedotin plus pembrolizumab and/or chemotherapy, versus chemotherapy alone, in untreated locally advanced or metastatic urothelial cancer. Ann Oncol 2020;31:S605-6.

21. Powles T, Park SH, Voog E, et al. Avelumab Maintenance Therapy for Advanced or Metastatic Urothelial Carcinoma. N Engl J Med 2020;383:1218-30.

22. Galsky MD, Mortazavi A, Milowsky MI, et al. Randomized Double-Blind Phase II Study of Maintenance Pembrolizumab Versus Placebo After First-Line Chemotherapy in Patients With Metastatic Urothelial Cancer. J Clin Oncol 2020;38:1797-806.

23. Rosenberg JE, Hoffman-Censits J, Powles T, et al. Atezolizumab in patients with locally advanced and metastatic urothelial carcinoma who have progressed following treatment with platinum-based chemotherapy: a single-arm, multicentre, phase 2 trial. Lancet 2016;387:1909-20.

24. Bellmunt J, de Wit R, Vaughn DJ, et al. Pembrolizumab as Second-Line Therapy for Advanced Urothelial Carcinoma. N Engl J Med 2017;376:1015-26.

25. Apolo AB, Infante JR, Balmanoukian A, et al. Avelumab, an Anti-Programmed Death-Ligand 1 Antibody, In Patients With Refractory Metastatic Urothelial Carcinoma: Results From a Multicenter, Phase Ib Study. J Clin Oncol 2017;35:2117-24. 
26. Powles T, O'Donnell PH, Massard C, et al. Efficacy and Safety of Durvalumab in Locally Advanced or Metastatic Urothelial Carcinoma: Updated Results From a Phase 1/2 Open-label Study. JAMA Oncol 2017;3:e172411.

27. Sharma P, Retz M, Siefker-Radtke A, et al. Nivolumab in metastatic urothelial carcinoma after platinum therapy (CheckMate 275): a multicentre, single-arm, phase 2 trial. Lancet Oncol 2017;18:312-22.

28. Fradet Y, Bellmunt J, Vaughn DJ, et al. Randomized phase III KEYNOTE-045 trial of pembrolizumab versus paclitaxel, docetaxel, or vinflunine in recurrent advanced urothelial cancer: results of $>2$ years of follow-up. Ann Oncol 2019;30:970-6.

29. Bellmunt J, Necchi A, De Wit R, et al. Pembrolizumab (pembro) versus investigator's choice of paclitaxel, docetaxel, or vinflunine in recurrent, advanced urothelial cancer (UC): 5 -year follow-up from the phase 3 KEYNOTE-045 trial. J Clin Oncol 2021;39:abstr 4532.

30. Powles T, Durán I, van der Heijden MS, et al. Atezolizumab versus chemotherapy in patients with platinum-treated locally advanced or metastatic urothelial carcinoma (IMvigor211): a multicentre, open-label, phase 3 randomised controlled trial. Lancet 2018;391:748-57.

31. Knowles MA, Hurst CD. Molecular biology of bladder cancer: new insights into pathogenesis and clinical diversity. Nat Rev Cancer 2015;15:25-41.

32. Li Q, Bagrodia A, Cha EK, et al. Prognostic Genetic Signatures in Upper Tract Urothelial Carcinoma. Curr Urol Rep 2016;17:12.

33. Loriot Y, Necchi A, Park SH, et al. Erdafitinib in Locally Advanced or Metastatic Urothelial Carcinoma. N Engl J Med 2019;381:338-48.

34. Moreno V, Loriot $\mathrm{Y}$, Valderrama BP, et al. Does escalation results from phase Ib/II Norse study of erdafitinib (ERDA)+ PD-1 inhibitor JNJ-63723283 (Cetrelimab [CET]) in patients (pts) with metastatic or locally advanced urothelial carcinoma (mUC) and selected fibroblast growth factor receptor (FGFR) gene alterations. J Clin Oncol 2020;38:abstr 511.

35. Bellmunt J, Théodore C, Demkov T, et al. Phase III trial of vinflunine plus best supportive care compared with best supportive care alone after a platinum-containing regimen in patients with advanced transitional cell carcinoma of the urothelial tract. J Clin Oncol 2009;27:4454-61.

36. Challita-Eid PM, Satpayev D, Yang P, et al. Enfortumab Vedotin Antibody-Drug Conjugate Targeting Nectin-4 Is a Highly Potent Therapeutic Agent in Multiple Preclinical Cancer Models. Cancer Res 2016;76:3003-13.
37. Rosenberg J, Sridhar SS, Zhang J, et al. EV-101: A Phase I Study of Single-Agent Enfortumab Vedotin in Patients With Nectin-4-Positive Solid Tumors, Including Metastatic Urothelial Carcinoma. J Clin Oncol 2020;38:1041-9.

38. Rosenberg JE, Heath EI, O'Donnell PH, et al. EV-201 Study: A single-arm, open-label, multicenter study of enfortumab vedotin for treatment of patients with locally advanced or metastatic urothelial cancer who previously received immune checkpoint inhibitor therapy. J Clin Oncol 2018;36:abstr TPS4590.

39. Rosenberg JE, O'Donnell PH, Balar AV, et al. Pivotal Trial of Enfortumab Vedotin in Urothelial Carcinoma After Platinum and Anti-Programmed Death 1/Programmed Death Ligand 1 Therapy. J Clin Oncol 2019;37:2592-600.

40. O'Donnell P, Galsky M D, Rosenberg JE, et al. 746P EV-201: Long-term results of enfortumab vedotin monotherapy for locally advanced or metastatic urothelial cancer previously treated with platinum and PD-1/PD-L1 inhibitors. Ann Oncol 2020;31:S579-80.

41. Powles T, Rosenberg JE, Sonpavde GP, et al. Enfortumab Vedotin in Previously Treated Advanced Urothelial Carcinoma. N Engl J Med 2021;384:1125-35.

42. Koshkin VS, Sun Y, Osterman CK, et al. 771P Efficacy of enfortumab vedotin in populations of interest among patients with advanced urothelial cancer. Ann Oncol 2020;31:S594.

43. Balar AV, McGregor BA, Rosenberg JE, et al. EV-201 Cohort 2: Enfortumab vedotin in cisplatin-ineligible patients with locally advanced or metastatic urothelial cancer who received prior PD-1/PD-L1 inhibitors. J Clin Oncol 2021;39:abstr 394.

44. Avellini C, Licini C, Lazzarini R, et al. The trophoblast cell surface antigen 2 and miR-125b axis in urothelial bladder cancer. Oncotarget 2017;8:58642-53.

45. Starodub AN, Ocean AJ, Shah MA, et al. First-inHuman Trial of a Novel Anti-Trop-2 Antibody-SN-38 Conjugate, Sacituzumab Govitecan, for the Treatment of Diverse Metastatic Solid Tumors. Clin Cancer Res 2015;21:3870-8.

46. Tagawa ST, Faltas B, Lam E, et al. Sacituzumab govitecan (IMMU-132) for patients with pretreated metastatic urothelial uancer (UC): interim results. Ann Oncol 2017;28:v301-2.

47. Tagawa ST, Faltas BM, Lam ET, et al. Sacituzumab govitecan (IMMU-132) in patients with previously treated metastatic urothelial cancer (mUC): Results from a phase I/II study. J Clin Oncol 2019;37:abstr 354. 
48. Tagawa ST, Petrylak DP, Grivas P, et al. TROPHY-u-01: A phase II open-label study of sacituzumab govitecan (IMMU-132) in patients with advanced urothelial cancer after progression on platinum-based chemotherapy and/ or anti-PD-1/PD-L1 checkpoint inhibitor therapy. J Clin Oncol 2019;37:abstr TPS3153.

49. Loriot Y, Balar AV, Petrylak DP, et al. LBA24 TROPHY-U-01 cohort 1 final results: A phase II study of sacituzumab govitecan (SG) in metastatic urothelial cancer (mUC) that has progressed after platinum (PLT) and checkpoint inhibitors (CPI). Ann Oncol 2020;31:S1156.

50. Petrylak DP, Tagawa ST, Jain RK, et al. Early results of TROPHY-U-01 Cohort 2: Sacituzumab govitecan (SG) in platinum-ineligible patients (pts) with metastatic urothelial cancer (mUC) who progressed after prior checkpoint inhibitor (CPI) therapy. J Clin Oncol 2020;38:abstr 5027.

51. Grivas P, Sternberg CN, Agarwal N, et al. 796TiP TROPHY-U-01 Cohort 3: Sacituzumab govitecan (SG) and pembrolizumab (pembro) in patients (pts) with progression or recurrence of metastatic urothelial cancer (mUC) after platinum (PLT)-based therapy. Ann Oncol 2020,31:S604-5.

52. Grivas P, Tagawa ST, Bellmunt J, et al. TROPiCS-04: Study of sacituzumab govitecan in metastatic or locally advanced unresectable urothelial cancer that has progressed after platinum and checkpoint inhibitor therapy. J Clin Oncol 2021;39:abstr TPS498.

53. Morrison K, Challita-Eid PM, Raitano A, et al. Development of ASG-15ME, a Novel Antibody-Drug Conjugate Targeting SLITRK6, a New Urothelial Cancer Biomarker. Mol Cancer Ther 2016;15:1301-10.

54. Petrylak D, Heath E, Sonpavde G, et al. Interim analysis of a phase I dose escalation trial of the antibody drug conjugate (ADC) AGS15E (ASG-15ME) in patients (Pts) with metastatic urothelial cancer (mUC). Ann Oncol 2016;27:vi269.

55. Hurvitz S A, Galsky MD, Shahidi J, et al. A phase Ib, multicenter, open-label study of the antibodydrug conjugate trastuzumab deruxtecan (DS-8201a) combination with nivolumab for advanced HER2expressing breast or urothelial cancer. Ann Oncol 2018;29:viii121.

56. Gong J, Shen L, Wang W, et al. Safety, pharmacokinetics and efficacy of RC48-ADC in a phase I study in patients with HER2-overexpression advanced solid cancer. J Clin Oncol 2018;36:abstr e16059.

57. Sheng X, Zhou AP, Yao X, et al. A phase II study of RC48-ADC in HER2-positive patients with locally advanced or metastatic urothelial carcinoma. J Clin Oncol 2019;37:abstr 4509.

58. Sheng X, Yan X, Wang L, et al. Open-label, Multicenter, Phase II Study of RC48-ADC, a HER2-Targeting Antibody-Drug Conjugate, in Patients with Locally Advanced or Metastatic Urothelial Carcinoma. Clin Cancer Res 2021;27:43-51.

59. Sheng X, He Z, Han W, et al. An open-label, single-arm, multicenter, phase II study of RC48-ADC to evaluate the efficacy and safety of subjects with HER2 overexpressing locally advanced or metastatic urothelial cancer (RC48-C009). J Clin Oncol 2021;39:abstr 4584.

60. Hinner MJ, Aiba RSB, Jaquin TJ, et al. Tumor-Localized Costimulatory T-Cell Engagement by the 4-1BB/HER2 Bispecific Antibody-Anticalin Fusion PRS-343. Clin Cancer Res 2019;25:5878-89.

61. Rothe C, Skerra A. Anticalin ${ }^{\circledR}$ Proteins as Therapeutic Agents in Human Diseases. BioDrugs 2018;32:233-43.

62. Ku G, Bendell JC, Tolcher AW, et al. $525 \mathrm{O}$ A phase I dose escalation study of PRS-343, a HER2/4-1BB bispecific molecule, in patients with HER2-positive malignancies. Ann Oncol 2020,31:S462-3.

63. Robertson AG, Kim J, Al-Ahmadie H, et al. Comprehensive Molecular Characterization of MuscleInvasive Bladder Cancer. Cell 2017;171:540-556.e25.

64. Grivas P, Loriot Y, Morales-Barrera R, et al. Efficacy and safety of rucaparib in previously treated, locally advanced or metastatic urothelial carcinoma from a phase 2, openlabel trial (ATLAS). BMC Cancer 2021;21:593.

65. Petrylak DP, de Wit R, Chi KN, et al. Ramucirumab plus docetaxel versus placebo plus docetaxel in patients with locally advanced or metastatic urothelial carcinoma after platinum-based therapy (RANGE): overall survival and updated results of a randomised, double-blind, phase 3 trial. Lancet Oncol 2020;21:105-20.

66. Apolo AB, Nadal R, Tomita Y, et al. Cabozantinib in patients with platinum-refractory metastatic urothelial carcinoma: an open-label, single-centre, phase 2 trial. Lancet Oncol 2020;21:1099-109.

67. Nadal R, Mortazavi A, Stein M, et al. Clinical efficacy of cabozantinib plus nivolumab (CaboNivo) and CaboNivo plus ipilimumab (CaboNivoIpi) in patients (pts) with chemotherapy-refractory metastatic Urothelial Carcinoma (mUC) either naïve (n) or refractory (r) to checkpoints inhibitors (CPI). J Clin Oncol 2018;36:abstr 4528.

68. Apolo AB, Nadal R, Girardi DM, et al. Phase I Study of Cabozantinib and Nivolumab Alone or With Ipilimumab for Advanced or Metastatic Urothelial 
Carcinoma and Other Genitourinary Tumors. J Clin Oncol 2020;38:3672-84.

69. Powles T, Kockx M, Rodriguez-Vida A, et al. Clinical efficacy and biomarker analysis of neoadjuvant atezolizumab in operable urothelial carcinoma in the ABACUS trial. Nat Med 2019;25:1706-14.

70. Necchi A, Anichini A, Raggi D, et al. Pembrolizumab as Neoadjuvant Therapy Before Radical Cystectomy in Patients With Muscle-Invasive Urothelial Bladder Carcinoma (PURE-01): An Open-Label, Single-Arm, Phase II Study. J Clin Oncol 2018;36:3353-60.

71. Hoimes CJ, Adra N, Fleming MT, et al. Phase Ib/II neoadjuvant $(\mathrm{N}-)$ pembrolizumab $(\mathrm{P})$ and chemotherapy for locally advanced urothelial cancer (laUC): Final results from the cisplatin (C)-eligible cohort of HCRN GU14188. J Clin Oncol 2020;38:abstr 5047.

72. Kaimakliotis HZ, Adra N, Kelly WK, et al. Phase II neoadjuvant (N-) gemcitabine (G) and pembrolizumab (P) for locally advanced urothelial cancer (laUC): Interim results from the cisplatin (C)-ineligible cohort of GU14188. J Clin Oncol 2020;38:abstr 5019.

73. van Dijk N, Gil-Jimenez A, Silina K, et al. Preoperative ipilimumab plus nivolumab in locoregionally advanced urothelial cancer: the NABUCCO trial. Nat Med 2020;26:1839-44.

74. Gupta S, Sonpavde G, Weight CJ, et al. Results from BLASST-1 (Bladder Cancer Signal Seeking Trial) of nivolumab, gemcitabine, and cisplatin in muscle invasive bladder cancer (MIBC) undergoing cystectomy. J Clin Oncol 2020;38:abstr 439.

75. Hussain MHA, Powles T, Albers P, et al. IMvigor010: Primary analysis from a phase III randomized study of adjuvant atezolizumab (atezo) versus observation (obs) in high-risk muscle-invasive urothelial carcinoma (MIUC). J Clin Oncol 2020;38:abstr 5000.

76. Bajorin DF, Witjes JA, Gschwend J, et al. First results from the phase 3 CheckMate 274 trial of adjuvant nivolumab vs placebo in patients who underwent radical surgery for high-risk muscle-invasive urothelial carcinoma (MIUC). J Clin Oncol 2021;39:abstr 391.

77. Balar AV, Kulkarni GS, Uchio EM, et al. Keynote 057: Phase II trial of Pembrolizumab (pembro) for patients (pts) with high-risk (HR) nonmuscle invasive bladder cancer (NMIBC) unresponsive to bacillus calmette-guérin (BCG). J Clin Oncol 2019;37:abstr 350.

78. Black PC, Tangen C, Singh P, et al. Phase II trial of atezolizumab in BCG-unresponsive non-muscle invasive bladder cancer: SWOG S1605 (NCT\# 02844816). J Clin Oncol 2020;38:abstr 5022.

79. Loriot Y, Balar AV, De Wit R, et al. Phase III study of first-line pembrolizumab (pembro) plus lenvatinib (len) in patients (pts) with advanced urothelial carcinoma (UC) ineligible for platinum-based chemotherapy: LEAP-011. J Clin Oncol 2020;38:abstr TPS597.

80. Galsky MD, Powles T, Dreicer R, et al. FIGHT-205: Phase II study of first-line pemigatinib (PEMI) plus pembrolizumab (PEMBRO) versus PEMI alone versus standard of care (SOC) for cisplatin (CIS) - Ineligible urothelial carcinoma (UC) with FGFR3 mutation or rearrangement. J Clin Oncol 2020;38:abstr TPS592.

81. Rosenberg JE, Gajate P, Morales-Barrera R, et al. Safety and preliminary efficacy of rogaratinib in combination with atezolizumab in a phase Ib/II study (FORT-2) of first-line treatment in cisplatin-ineligible patients (pts) with locally advanced or metastatic urothelial cancer (UC) and FGFR mRNA overexpression. J Clin Oncol 2020;38:abstr 5014.

82. Swami U, Grivas P, Pal SK, et al. Utilization of systemic therapy for treatment of advanced urothelial carcinoma: Lessons from real world experience. Cancer Treat Res Commun 2021;27:100325.
Cite this article as: Koshkin VS, Osbourne AS, Grivas P. Treatment options for advanced urothelial cancer after progression on chemotherapy and immune checkpoint inhibitors: a literature review. Transl Androl Urol 2021;10(10):4022-4035. doi: 10.21037/tau-21-123 